\title{
Shaping code in conjunction with DCT for PAPR reduction in multicarrier system
}

Younes Aimer ${ }^{1,2^{*}}$, Boubakar Seddik Bouazza ${ }^{1}$, Smail Bachir ${ }^{2}$ and Claude Duvanaud ${ }^{2}$

\author{
*Correspondence: \\ younes.aimer@univ-poitiers.fr \\ 2 XLIM Laboratory UMR-CNRS \\ 7252, Institute of Technology, \\ Angoulême-University \\ of Poitiers, 4 avenue de \\ Varsovie, 16021 Angoulême, \\ France \\ Full list of author information \\ is available at the end of the \\ article
}

\begin{abstract}
Nonlinear behavior and power efficiency of the Power Amplifier (PA) contradictorily depend on the input signal amplitude distribution. The transmitted signal in multi-carrier modulation exhibits high Peak-to-Average Power Ratio (PAPR) and large bandwidths, leading to the degradation of the radio link and additional generation out-of-band interferences, which degrade the quality of the transmission. Practical solutions exist, like a power back-off, but with unacceptable efficiency performances of the transmitter. This paper deals with efficiency and linearity improvement using a new PAPR reduction method based on the combination of Discrete Cosine Transform and shaping technique. The main principle is to determine an optimal coding scheme according to a trade-off between coding complexity and performance benefits in the presence of PA nonlinearities. Simulation and experimental results in the context of OFDM signal and using a 20 W-3.7 GHz Radio-Frequency Power Amplifier show an improvement on PAPR reduction of about $3.25 \mathrm{~dB}$. Also, the communication criteria like Bit Error Rate and Error Vector Magnitude are improved by about one decade and a half and $8 \%$, respectively.
\end{abstract}

Keywords: Nonlinear power amplifier, PAPR reduction, DCT technique, Shaping code, OFDM systems, WLAN 802.11y applications, 20 W-3.7 GHz RF-PA

\section{Introduction}

Orthogonal Frequency Division Multiplex (OFDM) is widely adopted in wireless communications to satisfy user requirements like high data rates, security, and mobility. Among their advantages, we can mention its high spectral efficiency as well as low complexity, implementation flexibility, and robustness against interferences and frequency selective fading [1]. However, this technique's major drawback is its high envelope fluctuations defined by the PAPR value [2]. Such a signal is degraded by the nonlinear characteristics of RF circuits, mainly the RF-PA (Radio-Frequency Power Amplifier) [3]. To limit signal distortions a solution consists in backing off input power away from the saturation region, but it's a costly solution reducing the electrical performances and the efficiency at the transmitter.

The objective of the PAPR reduction methods is to reduce signal fluctuations to operate near saturation region (high-efficiency region) while still respecting communication criteria like Bit Error Rate (BER), Error Vector Magnitude (EVM), data-rate, author(s) and the source, provide a link to the Creative Commons licence, and indicate if changes were made. The images or other third party material in this article are included in the article's Creative Commons licence, unless indicated otherwise in a credit line to the material. If material is not included in the article's Creative Commons licence and your intended use is not permitted by statutory regulation or exceeds the permitted use, you will need to obtain permission directly from the copyright holder. To view a copy of this licence, visit http:// creativecommons.org/licenses/by/4.0/. 
and frequency mask specifications. Various techniques have been proposed in the literature to mitigate the effect of this problem. Among them, we can cite methods like clipping, clipping and filtering [4-6], Active Constellation Extension (ACE) [8, 9], Tone Reservation (TR) [6, 7, 10-13], Selected Mapping (SLM) [14, 15], switching [16], coding methods [17, 18], Partial Transmit Sequence (PTS) $[9,19,20]$, interleaving [21, 22], and Discrete Cosine Transform (DCT) [23-25]. Those techniques can be classified into three classes [26]: coding techniques, adding signal techniques, and probabilistic techniques.

Table 1 presents the classification of the existing PAPR reduction techniques reviewed in the state of art. This classification is performed using different criteria like downward compatibility, average power increase, data rate degradation, complexity, and BER degradation [26].

Unlike the other PAPR reduction techniques, authors proposed coding techniques based on redundancy into the transmitted signal to avoid the high envelope fluctuations affecting the data rate and or power efficiency. Also, PAPR reduction in these methods leads to increased computational complexity, especially at the OFDM transmitter. Our objective here is to investigate the use of coding or pre-coding technique that improves the trade-off between the efficiency at the transmitter and the quality of the transmission in the presence of PA nonlinearities and memory effects. Precisely, our contribution can be classified among coding techniques:

- for systems supporting minor modifications at the transmitter and the receiver (not downward compatible),

- without an increase in the average power,

- without data rate degradation,

- with additional software implementation,

- and without BER and EVM degradation.

Table 1 Classification of PAPR reduction techniques

\begin{tabular}{|c|c|c|c|c|c|c|}
\hline & & Communicatio & ns Criteria & & & \\
\hline PAPR reductio & echniques & Downward & Average & Data rate & Complexity & BER \\
\hline Coding tech- & Trellis shaping & $x$ & $x$ & $\sqrt{ }$ & $\sqrt{ }$ & $x$ \\
\hline & Block coding & $x$ & $x$ & $\sqrt{ }$ & $x$ & $x$ \\
\hline Adding signal & TR & $\sqrt{ }$ & $\sqrt{ }$ & $x$ & $\sqrt{ }$ & $x$ \\
\hline & TR (IFFT/DCT) & $\sqrt{ }$ & $\sqrt{ }$ & $x$ & $\sqrt{ }$ & $x$ \\
\hline & Clipping & $\sqrt{ }$ & $\sqrt{ }$ & $x$ & $x$ & $\sqrt{ }$ \\
\hline & Clipping-filtering & $\sqrt{ }$ & $\sqrt{ }$ & $x$ & $x$ & $x$ \\
\hline & ACE & $\sqrt{ }$ & $\sqrt{ }$ & $\sqrt{ }$ & $x$ & $\sqrt{ }$ \\
\hline Probabilistic & Interleaving & $\sqrt{ }$ & $x$ & $x$ & $x$ & $x$ \\
\hline techniques & Switching & $\sqrt{ }$ & $x$ & $\sqrt{ }$ & $\sqrt{ }$ & $\sqrt{ }$ \\
\hline & SLM & $\times$ & $x$ & $\sqrt{ }$ & $\sqrt{ }$ & $\sqrt{ }$ \\
\hline & SLM blind & $\sqrt{ }$ & $x$ & $x$ & $\sqrt{ }$ & $x$ \\
\hline & SLM-DCT & $\sqrt{ }$ & $x$ & $\sqrt{ }$ & $\sqrt{ }$ & $\sqrt{ }$ \\
\hline & PTS & $x$ & $x$ & $\sqrt{ }$ & $\sqrt{ }$ & $\sqrt{ }$ \\
\hline
\end{tabular}


In this work, we focused on the coding techniques that present a viable solution to improve service quality in digital transmission. As for interleaving [21, 22], it is possible to act on the input signal, to reduce its peaks. The main idea is the implementation of pre-coding techniques like the DCT $[18,25]$, generally used in an image or video compression and wellknown for its performances in PAPR reduction [23, 24]. However, DCT theoretically has no direct influence on the transmission quality, and this is why we will investigate its conjunction with the shaping code, for its properties in the control and the conversion of the signal distribution [27]. This coding adds binary redundancy and makes it possible to shape the constellation to minimize its average energy and improve the system's performance [27, 29]. This point is crucial because the increase in average power energy is the main disadvantage of other efficient techniques like TR.

After a brief overview of the OFDM system and the PAPR problem, we will describe the proposed method in Sect. 2 by explaining its principle, advantages, and simulation results for WLAN 802.11y standard. Section 3 will be devoted to the preliminary evaluation of the proposed technique in the presence of a nonlinear memory polynomial model. Finally, we will provide experimental results and a comparative study using a class-AB PA (3.4 to 3.8 $\mathrm{GHz}, 32.5 \mathrm{dBm}$ ), and we will conclude.

\section{Methods/experimental}

\subsection{OFDM system and problem position}

In discrete-time, an OFDM symbol $x_{n}$ is obtained by the Inverse Fast Fourier Transform (IFFT) on the $N$ mapped symbols such as:

$$
x_{n}=\frac{1}{\sqrt{N}} \sum_{k=0}^{N-1} X_{k} e^{\frac{j 2 \pi n k}{N}} \quad 0 \leq n \leq N-1
$$

where $X_{k}, k=0,1, \ldots, N-1$ are the mapped symbols, usually modulated by a Quadrature Amplitude Modulation (QAM), and $k$ is the discrete-time index.

It is known that OFDM modulation is a particular case of multi-carrier transmission, where a single data stream is transmitted over a number of lower rate orthogonal sub-carriers. Also, among its advantages we can cite the high spectral distribution and efficiency, high data rate, robustness to the multi-path channel, and low complexity of implementation [1]. However, as shown in relation (1), the resulting signal is the superposition of $N$ independent narrow-band channels that can generate constructive and or destructive sums, and consequently, high envelope fluctuations. High instantaneous peaks limit the nonlinear RF-PA to operate at lower average power, known as linear region, which degrades transmitter efficiency. Improving the power efficiency implies the reduction of fluctuations to maintain a higher average power without signal degradation.

The PAPR of a signal, describing the ratio of the maximum instantaneous power and its average power for each OFDM symbol, is defined as [28]:

$$
\text { PAPR }_{\mathrm{dB}}=10 \cdot \log \left(\frac{P_{\text {Peak }}}{P_{\text {Average }}}\right)=10 \cdot \log \left(\frac{\underset{0 \leq n \leq N-1}{\max }\left|x_{n}\right|^{2}}{E\left[\left|x_{n}\right|^{2}\right]}\right)
$$


where $P_{\text {Peak }}$ represents peak output power, $P_{\text {Average }}$ means average output power. $E[\cdot]$ denotes the expected value operation and $x$ the vector of OFDM samples in timedomain such as $x=\left[x_{0}, x_{1}, \ldots, x_{(N-1)}\right]^{T}$.

\subsection{Proposed method}

\subsubsection{Discrete Cosine transform (DCT)}

The DCT is widely used in signal and image processing. It separates the signal, like a sound or image, into different sub-bands with different weights according to the spectrum, and consequently, to the signal quality. Like DFT (Discrete Fourier Transform), it appears from the temporal or spatial domain to the frequency domain [30]. The literature presents a multitude of DCTs transforms, like uni-dimensional DCT (DCT-I) [31], two-dimensional DCT (DCT-II) [32] and three-dimensional DCT (DCT-III) [33]. For example, the DCT-II is introduced to transform the data sequence from an image to obtain a high inter-pixels correlation and reduce the spatial redundancy [30]. Differentiating the spectral sub-bands is highly important concerning the image visual quality in compression process. In our case, we study the DCT for its properties on spectral compaction and the reduction of data sequences auto-correlation, without increasing the power average of the original signal [25].

\section{Basic principle:}

The DCT is very similar to the DFT, and its basic principle is presented in Fig. 1

At the end of the DCT, each output sample of rank $m$, denoted $S_{m}^{D C T}$, will be calculated from vector $C$ composed of $N$ modulated symbols $C_{k}$ with $k=0 \ldots N-1$ and expressed such as:

$$
S_{m}^{\mathrm{DCT}}=\sqrt{\frac{D}{N}} \sum_{k=0}^{N-1} C_{k} \cos \left(\frac{\pi}{N} \frac{(2 k+1) m}{2}\right)
$$

where $D=\left\{\begin{array}{ll}1 & \text { if } m=0 \\ 2 & \text { if } m \neq 0\end{array} \quad\right.$ for $\quad k, n=0,1, \ldots, N-1$ The output $S_{m}^{\mathrm{DCT}}$ of the DCT block is a set of coefficients corresponding to a DCT basis function (cosine functions with increasing frequencies).

At the receiver, each of these basis functions is then multiplied by the corresponding coefficients, and the sum of those values gives the reconstruction of the original

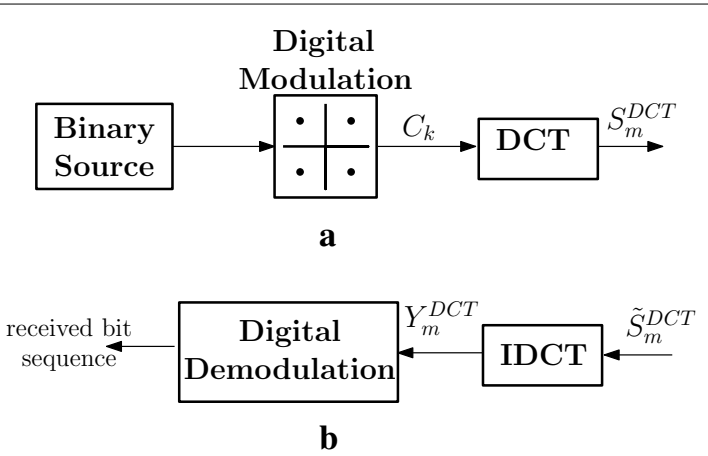

Fig. 1 Block scheme of DCT a transmitter, $\mathbf{b}$ receiver 


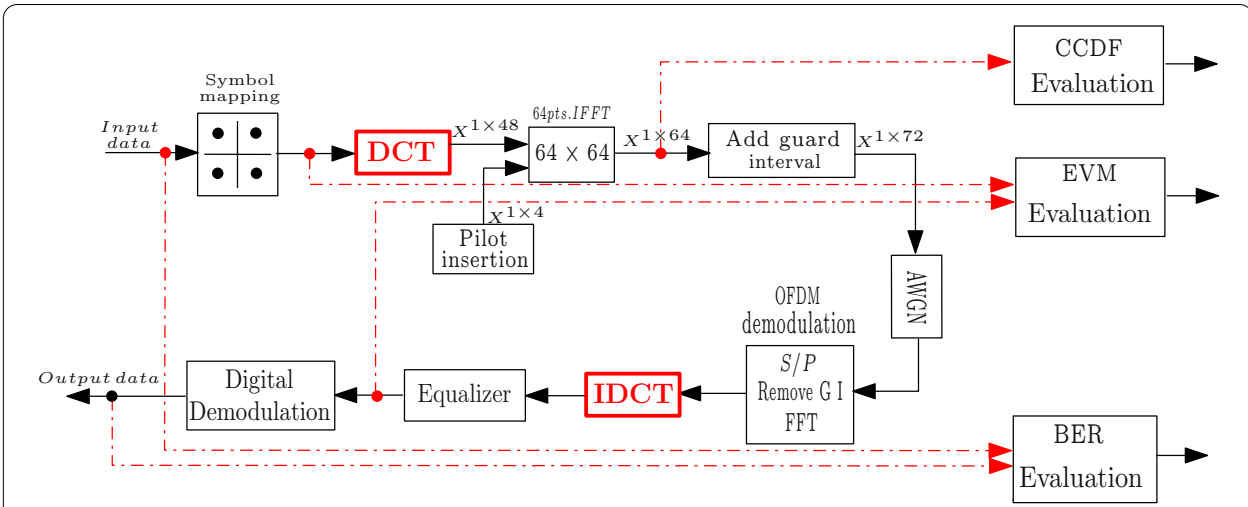

Fig. 2 Simulation block with the DCT (WLAN 802.11y standard)

Table 2 Simulation parameters

\begin{tabular}{ll}
\hline Parameter & Value \\
\hline Num. of sub-carriers & 64 (48 data, 4 pilots \\
& and 12 null sub- \\
carriers $)$
\end{tabular}

signal. This process is carried out with the use of the Inverse Discrete Cosine Transform (IDCT) $[18,25]$.

\section{Evaluation of the DCT in simulation:}

In order to evaluate the performances of the DCT and its impact in the quality of transmission, we carried out a series of simulations with and without DCT for the WLAN IEEE.802.11y standard, through the block scheme presented in Fig. 2.

The simulation block is implemented in MATLAB software platform according to the parameters presented in Table 2. The used standard adopts OFDM technique for UpLink (UL) and Down-Link (DL) with a carrier frequency of $3.7 \mathrm{GHz}$.

The method evaluation is performed as follows: preliminary results allow performances evaluation in term of PAPR reduction at the transmitter using Complementary Cumulative Distribution Function (CCDF) and using communication criteria like EVM and BER.

The CCDF of the PAPR is one of the most frequent parameters for PAPR reduction techniques. It denotes the probability that the PAPR of a data block exceeds a given threshold $\mathrm{PAPR}_{0}$ and is expressed as:

$$
\mathrm{CCDF}=\mathrm{PR}\left(\mathrm{PAPR}>\mathrm{PAPR}_{0}\right)
$$

where $\mathrm{PR}(\cdot)$ is the probability function. 


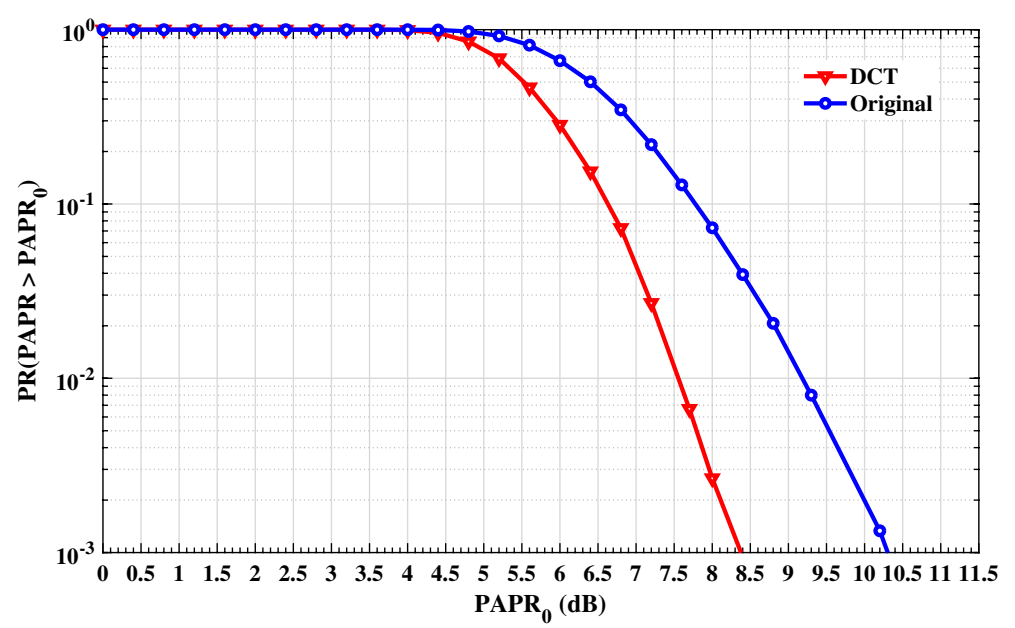

Fig. 3 CCDF curves

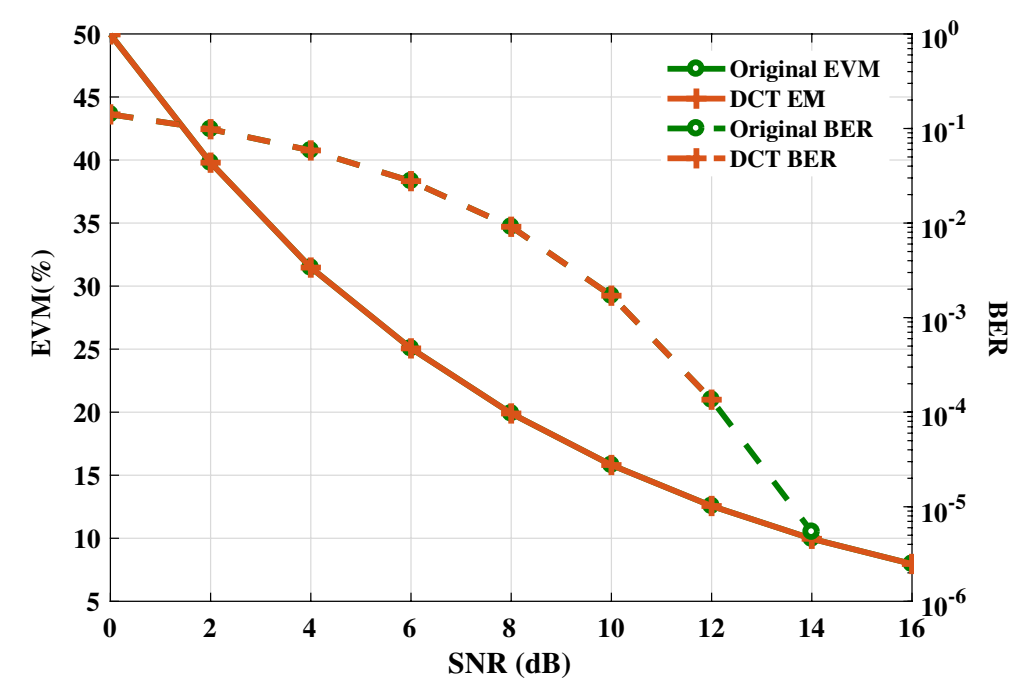

Fig. 4 EVM and BER curves

Figure 3 presents the CCDF of the obtained OFDM signal with and without the DCT. According to these curves, it can be seen that the DCT technique makes it possible to improve the performance in terms of PAPR reduction by approximately $2 \mathrm{~dB}$ at $10^{-3}$ of probability, in comparison with the original signal.

These results confirm the relationship between the auto-correlation reduction theory of signals after DCT and the reduction of fluctuations.

To evaluate the quality of transmission in the presence of Additive White Gaussian Noise (AWGN) channel, we present in Fig. 4 the EVM and BER curves versus SNR (Signal-to-Noise Ratio), respectively, with and without DCT.

From the obtained results in terms of EVM and BER, we can see clearly that the DCT technique does not introduce any modification on these two criteria: the EVM 
and BER are similar to the original signal. The impact of the PAPR reduction is not perceptible in the absence of the amplifier nonlinearities.

We can conclude that the use of the DCT improves the PAPR reduction without any amelioration of transmission quality (in terms of EVM and BER) in the presence of AWGN channel. To deal with this inconvenient, in the next section, we suggest a new scheme of PAPR reduction, to improve performances.

\subsubsection{Shaping technique}

The shaping technique is one form of constellation shaping, usually used to control the transmission of data symbols and convert the signal distribution into Gaussian [27]. The main idea to reduce the average constellation power and power consumption is to transmit digital symbols with low energy more frequently. The achieved symbols will be composed of bits with unequal probabilities, and this will be used to shape the QAM constellation points. The signal constellation is then divided into sub-constellations, each having its own energy, as shown in Fig. 5 for a 16-QAM example.

As illustrated in Fig. 5 and using the basic block diagram of the shaping technique described in Fig. 6, where S.C referred to as a Shaping Code, the constellation will be partitioned into four sub-constellations (low energy for region 1, medium energy for 2 and 3 , and high energy for region 4).

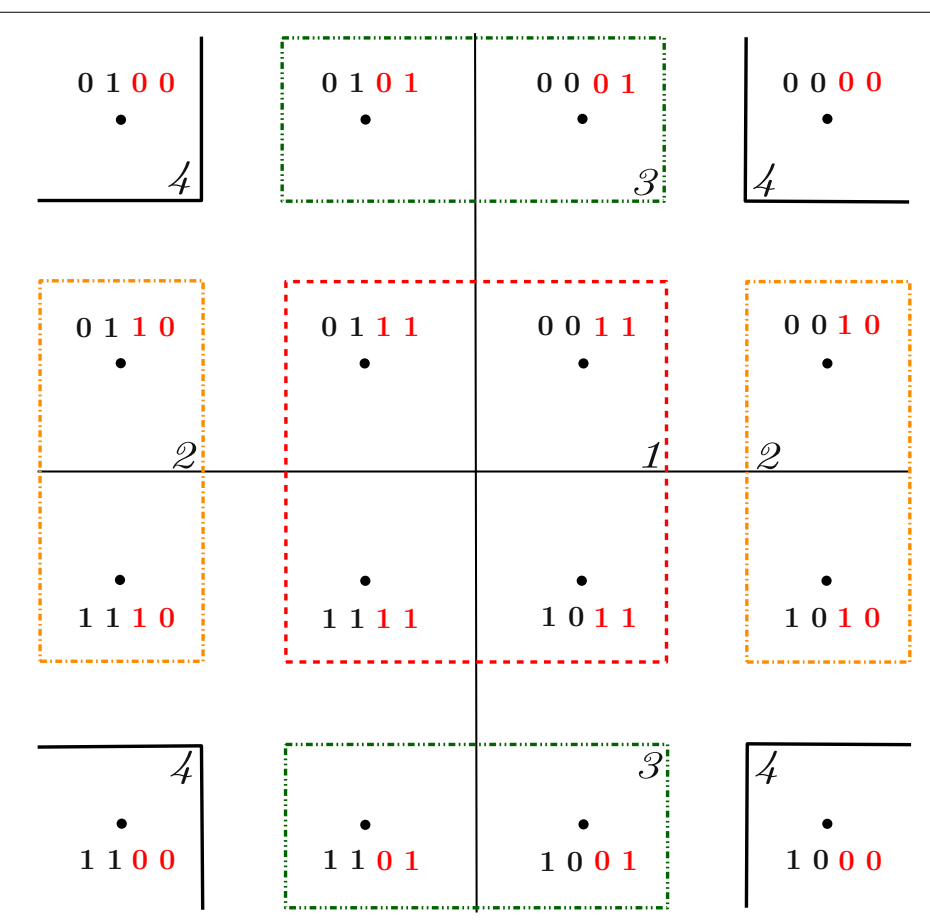

Sub-constellation 1:Symbols with low energy

Sub-constellation 2/Sub-constellation 3:Symbols with average energy

Sub-constellation 4:Symbols with high energy

XX: Shaping bits

Fig. 5 Constellation mapping for shaping technique (16-QAM) 


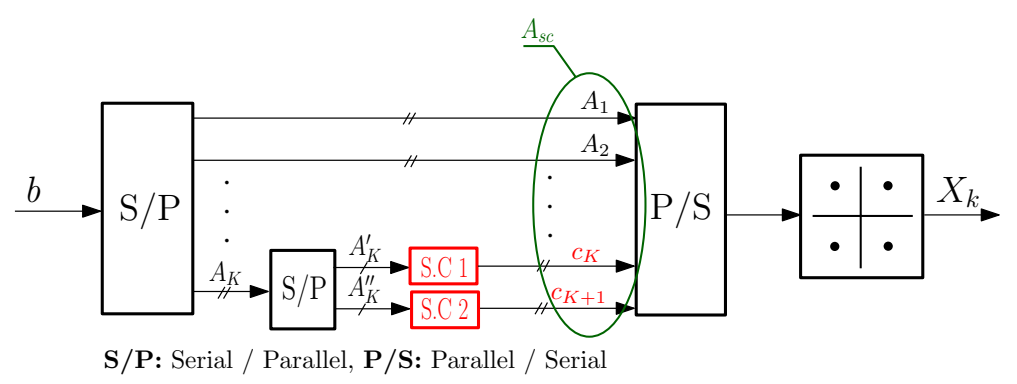

Fig. 6 General block scheme of the shaping technique, $K=3$ in the case of 16-QAM modulation

Table 3 Proposed shaping code $\left(k_{s}=2, n_{s}=4\right)$

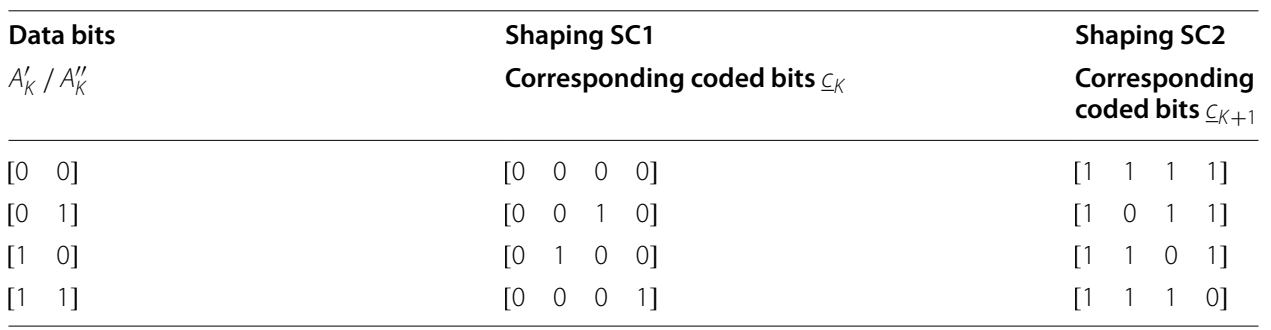

This division induces the same format for the two LSB (Least Significant Bits) in each region (see the two LSB in red color on Fig. 5). For example, symbols in region 1 use the binary format "xx11", in region 2 "xx10", in region 3 "xx01" and the format "xx00" in region 4 . Thus, the binary words of each symbol $A_{s c}$ are located in its region using the 2 $\operatorname{LSB}\left(c_{k}, c_{k+1}\right)$. Its position in the specified region is according to its 2 MSB (Most Significant Bits) $\left(\left(A_{1}, A_{2}\right)\right)$. The aim of our study is to generate additional bits to prioritize symbols with low and medium energy.

For example, Table 3 presents the coding Table of the used shaping codes (SC1 and SC2) for average power reduction of the transmitted signal. The coding rate is $R s=k_{s} / n_{s}=1 / 2$, where $k_{s}$ and $n_{s}$ are the numbers of input and output bits of the encoder. This shaping generates couples of LSB bits $\left[c_{K} c_{K+1}\right]$ that concentrate the mapping symbols in the medium and low energy sub-constellations (Fig. 5). For example, the first line of Table 3 indicates that when $\left[A_{K}^{\prime} A_{K}^{\prime \prime}\right]=\left[\begin{array}{llll}0 & 0 & 0 & 0\end{array}\right]$, the generated sequence $\left[c_{K} c_{K+1}\right]$ are all equal to [0 1], which systematically locate the symbol in the sub-constellation 3 (medium region). Note that $c_{K}$ and $c_{K+1}$ are the outputs of SC1 and SC2 blocks and $\left[c_{K} c_{K+1}\right]$ correspond to the 2 LSB of the modulated symbol.

As presented in Fig. 6, the proposed shaping technique in the general case on NOFDM sub-carriers, with M-ary QAM modulation, can be expressed as the following steps:

Step 1: First, the bits sequence $\underline{b}$ is partitioned into $N$ blocks of $K=\log _{2}(M)-1$ bits, i.e., $b=\left[b_{0} b_{1} \ldots b_{N-1}\right]$ and $A_{i}=\left[b_{i, 0} b_{i, 1} \ldots b_{i, N-1}\right], i=0,1, \ldots, K$.

where $M$ presents the constellation size, and $K$ is the bit number per symbol.

Step 2: The sequence $A_{K}=\left[b_{K, 0} b_{K, 1} \ldots b_{K, N-1}\right]$ is then further divided into the form $A_{K}=\left(A_{K}^{\prime} A_{K}^{\prime \prime}\right)$ using serial/parallel block.

Step 3: Shaping code blocks SC1 and SC2 introduce, according to Table 3, one-bit redundancy per QAM symbols to obtain the coded sequence $A_{s c}=\left(A_{1} A_{2} \ldots c_{K} c_{K+1}\right)$. 


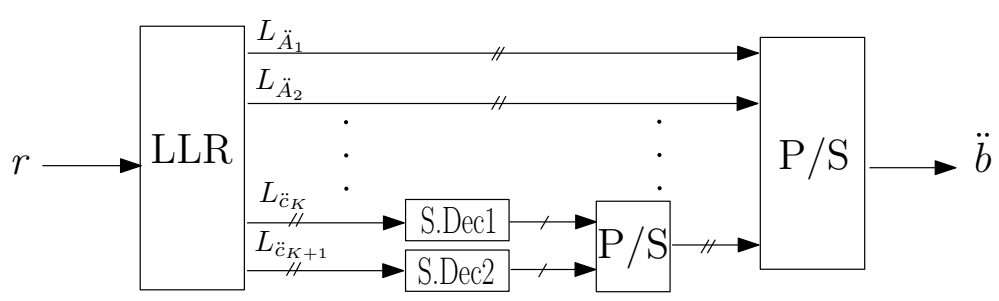

Fig. 7 Shaping technique block at the receiver

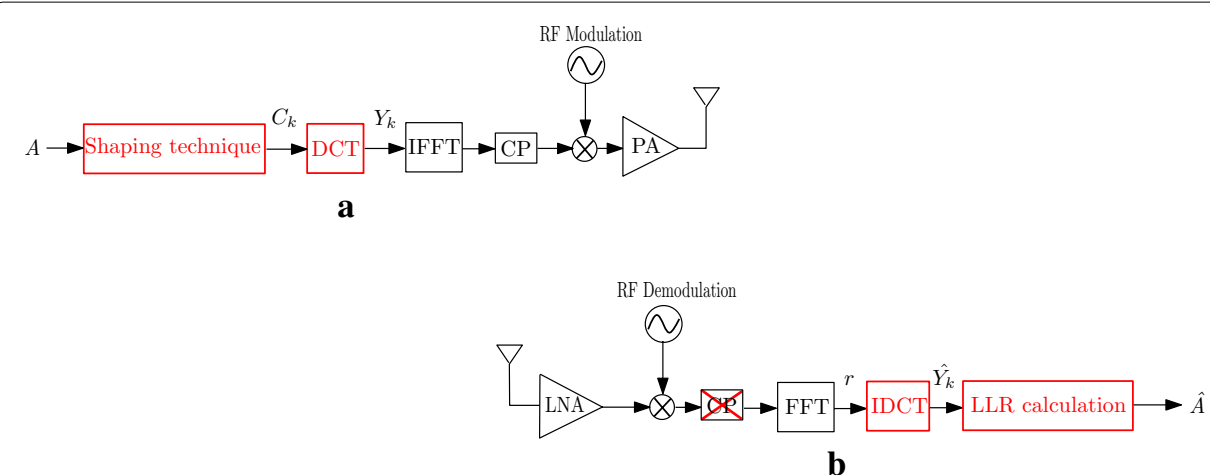

Fig. 8 The proposed OFDM system with shaping and DCT techniques a transmitter, $\mathbf{b}$ receiver

Step 4: Output sequences of the shaping technique are mapped in QAM before transmission.

At the receiver (Fig. 7), the output LLR (Logarithm Likelihood Ratio) block is used, which produces $K+1$ vectors $L=\left(L_{\ddot{A}_{1}} L_{\ddot{A}_{2}} \ldots L_{\ddot{c}_{K}} L_{\ddot{c}_{K+1}}\right)$.

The received bits $L_{\ddot{c}_{K}}, L_{\ddot{c}_{K+1}}$ will be decoded using the MAP (Maximum A posteriori Probability) algorithm to generate the estimated bits. The LLR's expression is given in [29] as:

$$
L\left(\hat{a}_{q, 1}\right)=\ln \left(\frac{\sum_{c_{K} \in \phi_{q}^{1}} \exp \left(\sum_{i=1}^{n_{s}} t_{i} L\left(\ddot{c}_{i_{, K}}\right)\right)}{\sum_{c_{K} \in \phi_{q}^{0}} \exp \left(\sum_{i=1}^{n_{s}} t_{i} L\left(\ddot{c}_{i, K}\right)\right)}\right)
$$

where $\phi_{q}^{t}\left(t \in\{0,1\}, q \in\left\{1, \ldots, k_{s}\right\}\right)$ denotes the set of all codewords $c_{K}$ obtained after shaping code and $t_{i}$ is the value of the $i$ th bit in the codeword $c_{K}$ under consideration.

Figure 8 presents the proposed method, using a block coding for shaping code, combined with DCT at the emission and IDCT with the LLR algorithm at the receiver. The main idea is to combine the advantages of these two techniques to improve the PAPR reduction and maintain the same average power than the original signal. 


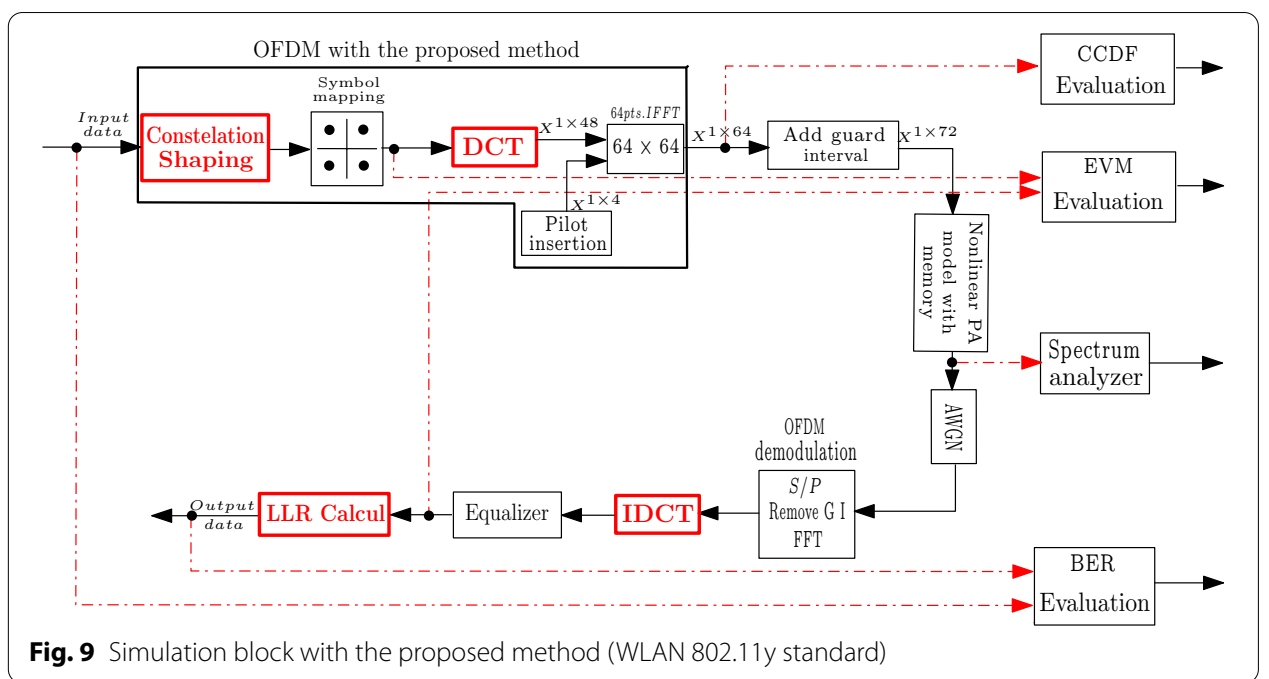

Fig. 9 Simulation block with the proposed method (WLAN 802.11y standard)

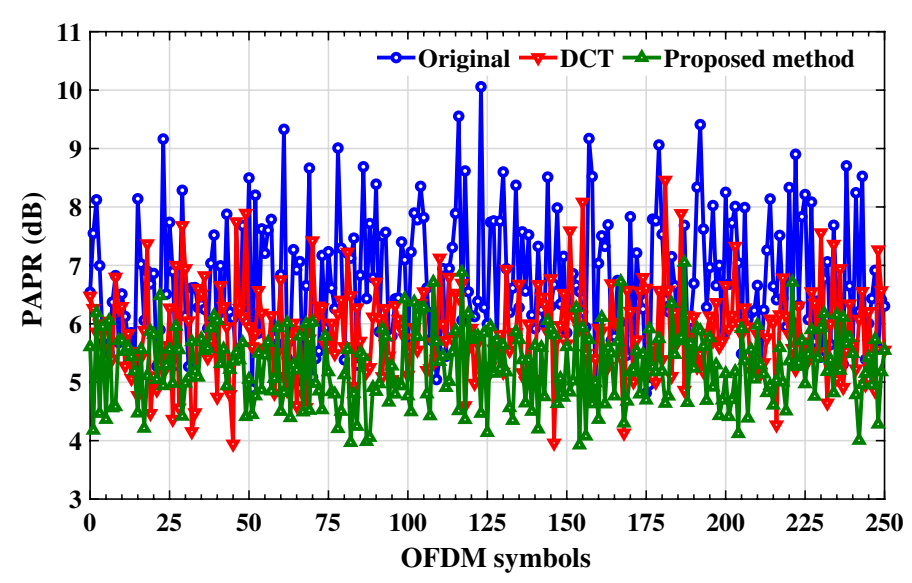

Fig. 10 Comparison of PAPR values versus OFDM symbols

\section{Results and discussion}

\subsection{Simulation results}

The proposed method is evaluated through simulations for the WLAN 802.11y standard and according to the system presented in Fig. 9. Simulation parameter is given in Table 2.

The evaluation method is performed as follows: preliminary results to study the performances in term of PAPR reduction at the transmitter using CCDF. Subsequently, we will evaluate the proposed method in the presence of nonlinear PA model with memory and a noisy channel using communication criteria such as EVM, BER and ACPR (Adjacent Channel Power Ratio). 


\subsubsection{PAPR reduction}

Figure 10 presents the variation of the PAPR value as a function of OFDM symbols. For each OFDM symbol, we evaluate the PAPR in the case of the original signal (blue curve), DCT only (red curve) and the proposed method combining shaping technique and DCT (green curve).

As we can see, the proposed method achieves more improvement than the DCT only, and the fluctuations in the transmitted signal are significantly reduced compared to the original signal.

Figure 11 presents the comparison of the CCDF curves. It can be seen that the CCDF curves of the original signal and the shaping code are similar. There was no reduction of PAPR when we used shaping code only. The DCT succeeds in improving the results, and its combination with shaping technique allows additional improvement. For example and for the probability of $10^{-3}$, the proposed method allows more than $3 \mathrm{~dB}$ improvement of PAPR reduction.

\subsubsection{Performance evaluation in the presence of nonlinear PA model with memory}

Power amplifiers are nonlinear devices modeled usually by their static amplitude and phase characteristics (AM/AM and AM/PM) and dynamic phenomena like short and long memory [35].

For simulations, we use a memory polynomial model obtained through experimental studies of a commercial $2 \mathrm{~W}$ class AB $2.2 \mathrm{GHz}$, InGaP power amplifier from RF Micro Devices. PA identification allows an accurate model with nonlinear order $P_{P A}=5$ and memory length of $M_{P A}=1$.

The input complex envelope $u(t)$ to output complex envelope $y(t)$ relationship is expressed as:

$$
y(t)=\sum_{m=0}^{M_{P A}} \sum_{p=0}^{P_{P A}} \alpha_{m, 2 p+1} \cdot|u(t-m)|^{2 p} . u(t-m)
$$

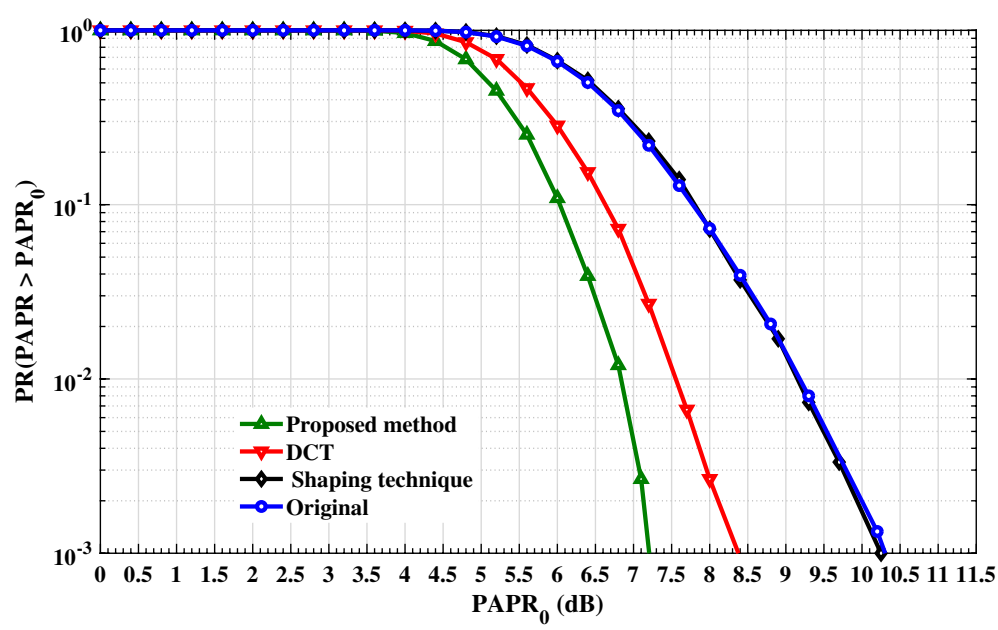

Fig. 11 Comparison of CCDF curves 
Table 4 PA Model parameters

\begin{tabular}{ll}
\hline Values & \\
\hline$\alpha_{0.1}$ & $-3.1+2.93 j$ \\
$\alpha_{1,1}$ & $2.86 \cdot 10^{-1}+2.19 \cdot 10^{-1} j$ \\
$\alpha_{0,3}$ & $5.15 \cdot 10^{-2}-4.2 \cdot 10^{-2} j$ \\
$\alpha_{1,3}$ & $-3.49 \cdot 10^{-4}+2.75 \cdot 10^{-4} j$ \\
$\alpha_{0,5}$ & $-4.93 \cdot 10^{-5}+1.37 \cdot 10^{-5} j$ \\
$\alpha_{1,5}$ & $7.58 \cdot 10^{-3}-4.34 \cdot 10^{-3} j$ \\
\hline
\end{tabular}

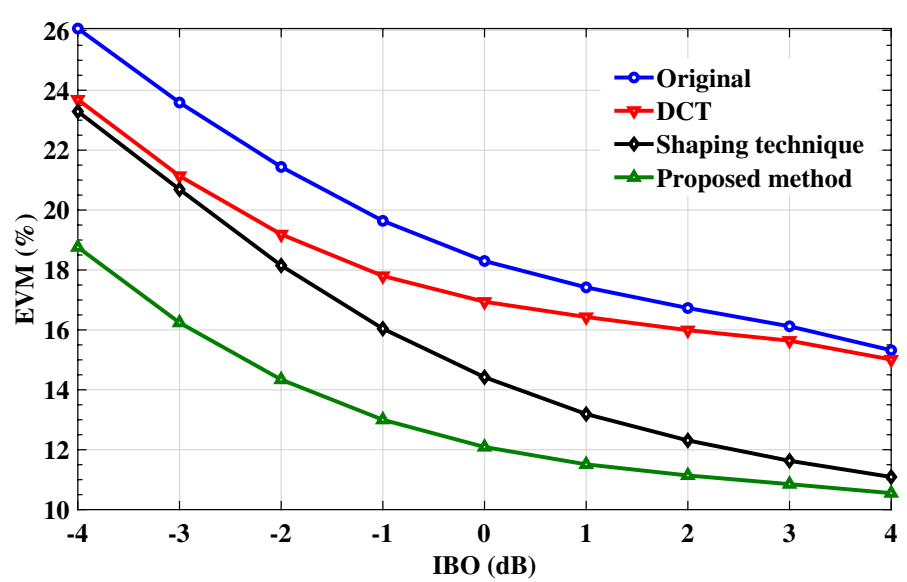

Fig. 12 Comparison of EVM versus IBO $(S N R=12 d B)$

where $\alpha_{m, 2 p+1}$ are complex coefficients estimated using the Least-Square algorithm on multi-carrier signals with $20 \mathrm{MHz}$ bandwidth. Table 4 gives the obtained model parameters.

All results are given versus power Input Back-Off (IBO) which defines the difference between the input power $P_{\text {in }}$ of the operating point and the $1 \mathrm{~dB}$ compression point $P_{i n}^{1 d B}$, such as :

$$
\mathrm{IBO}(\mathrm{dB})=P_{\text {in }}(\mathrm{dBm})-P_{\text {in }}^{1 d B}(\mathrm{dBm})
$$

For the used PA, $P_{i n}^{1 d B}$ corresponds to $20 \mathrm{dBm}$ of input power.

According to the simulation setup presented in Fig. 9, EVM and BER curves versus IBO with a noisy channel of SNR $=12 \mathrm{~dB}$ are shown in Figs. 12, 13, using DCT only (red curve), shaping technique only (black curve) and the proposed method (green curve).

Figures 12 and 13 show that using only the DCT technique gives an insufficient improvement to the system in the linear region $(\mathrm{IBO}>0 \mathrm{~dB})$, while the shaping only allows an improvement of about 4\% of EVM and more than one decade of BER in this region. In the nonlinear region $(\mathrm{IBO}<0 \mathrm{~dB})$, shaping still gives better results than the DCT except at high saturation $(\mathrm{IBO}=-4 \mathrm{~dB})$, where the results are substantially similar. The proposed method improves the EVM significantly in the nonlinear region, while the BER remains like shaping only. In fact, the EVM criterion evaluates the system linearity (e.g., in-band distortion), while the BER gives information on the decoding ability. 


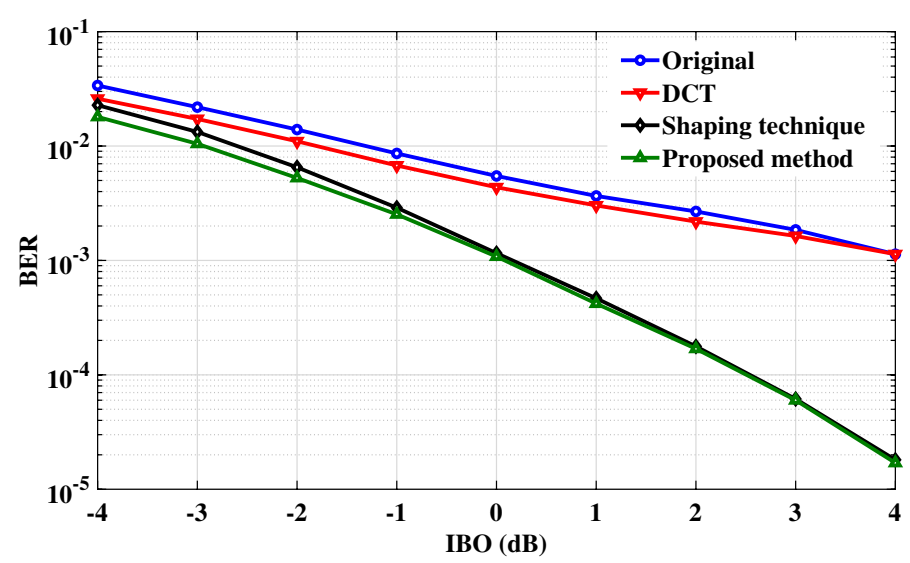

Fig. 13 Comparison of $B E R$ curves versus $I B O(S N R=12 d B)$

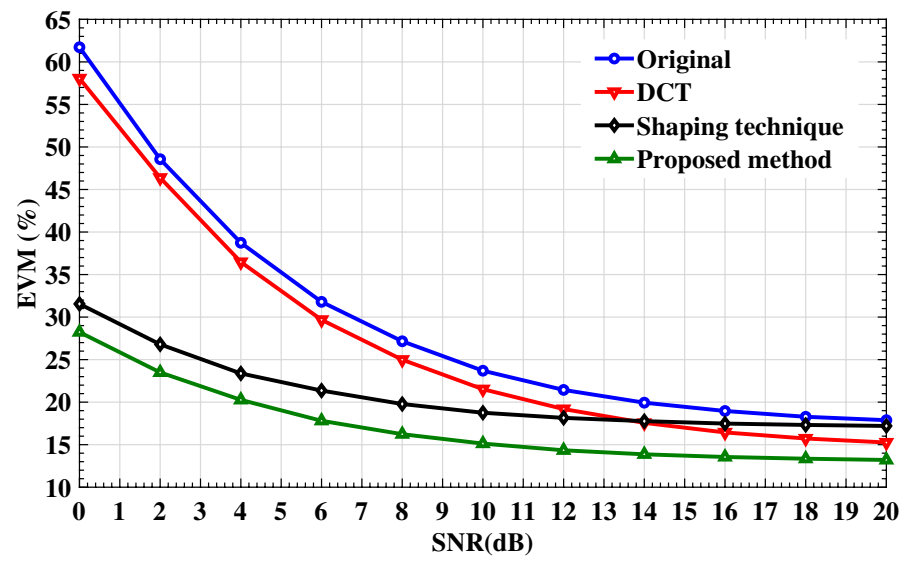

Fig. 14 EVM Curves versus SNR for Gaussian channels $(I B O=-2 d B)$

Compared to the original signal, the proposed method allows an improvement of 7.5\% for EVM and one decade and a half for BER. We can read the EVM curve differently in term of linearity such as: for the same quality (same EVM), the proposed method makes it possible to obtain a maximal gain of $6 \mathrm{~dB}$ in IBO. For an illustration of the gain in linearity, Fig. 12 shows that an EVM of $16 \%$ can be reached at IBO of $3 \mathrm{~dB}$ for original signal and $-3 \mathrm{~dB}$ for the proposed technique. In this case, the power amplifier can be used in its nonlinear region, which improves the transmitter efficiency, without loss in quality of service.

Figures 14 and 15 present, respectively, the EVM and the BER curves of the received signal versus SNR in the nonlinear region corresponding to $\mathrm{IBO}=-2 \mathrm{~dB}$.

As previous results, we can observe that using the shaping code with the DCT allows significant EVM performances, compared to the DCT method, which has almost the same behavior as the original signal.

Constellations in Fig. 16 for an IBO of -2dB, i.e., PA operating in the nonlinear region, and noisy channel with SNR equal to $12 \mathrm{~dB}$ show that applying the proposed method reduces the symbols dispersion despite PA nonlinearities. We can notice the shaping 


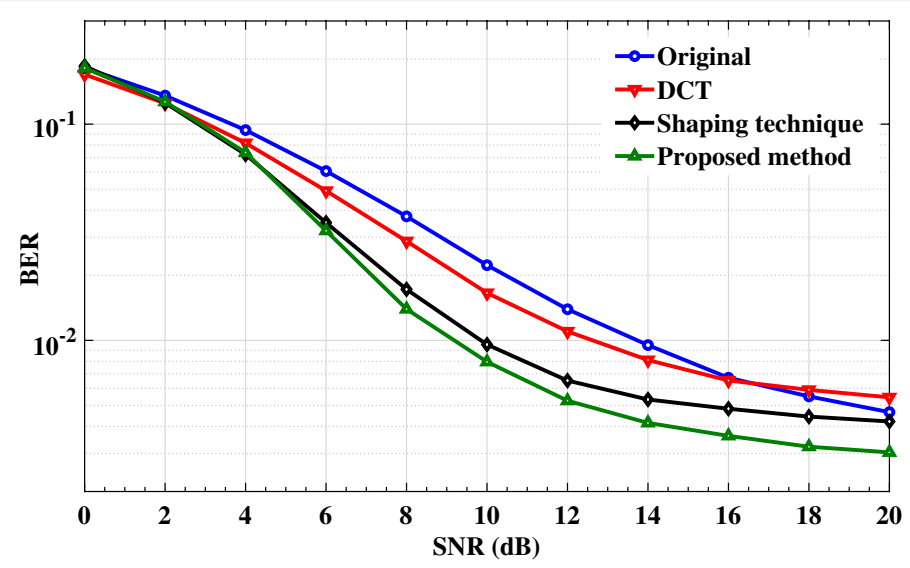

Fig. 15 BER Curves versus SNR for Gaussian channels (IBO $=-2 \mathrm{~dB})$
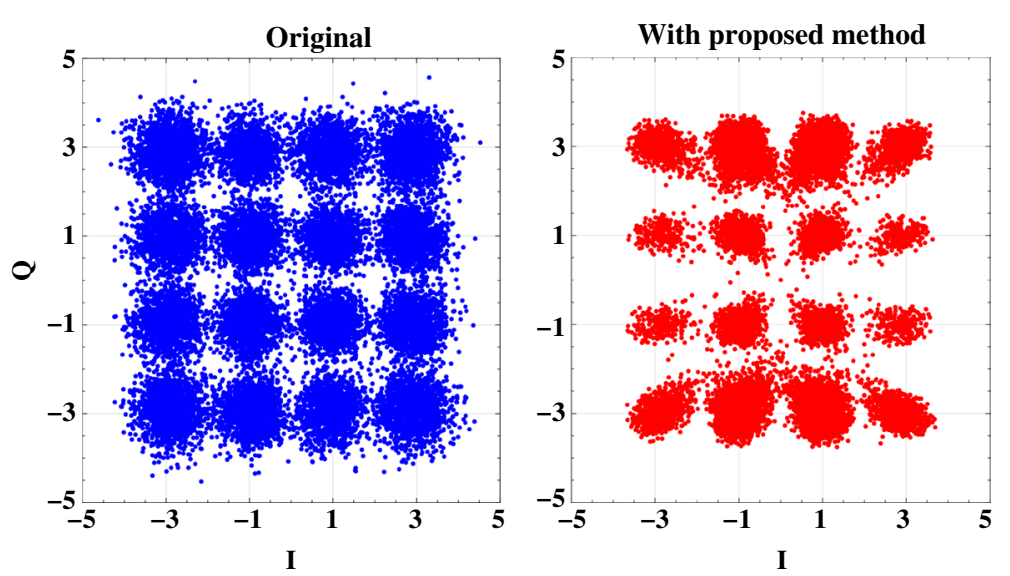

Fig. 16 Constellation with and without PAPR reduction $(\mathrm{IBO}=-2 \mathrm{~dB})$

Table 5 ACPR results

\begin{tabular}{|c|c|c|c|c|c|c|}
\hline \multirow[b]{2}{*}{$\mathrm{IBO}(\mathrm{dB})$} & \multicolumn{2}{|l|}{ Original } & \multicolumn{2}{|l|}{ DCT } & \multicolumn{2}{|c|}{ Proposed method } \\
\hline & $\mathrm{ACPR}_{\mathrm{dB}}^{L}$ & $\mathbf{A C P R}_{\mathrm{dB}}^{U}$ & $\mathrm{ACPR}_{\mathrm{dB}}^{L}$ & $\mathbf{A C P R}_{\mathrm{dB}}^{U}$ & $\mathrm{ACPR}_{\mathrm{dB}}^{L}$ & $\mathrm{ACPR}_{\mathrm{dB}}^{U}$ \\
\hline$-4 \mathrm{~dB}$ & -21.67 & -21.78 & -22.39 & -22.46 & -23.01 & -22.93 \\
\hline$-2 \mathrm{~dB}$ & -24.71 & -24.86 & -25.86 & -25.95 & -26.50 & -26.61 \\
\hline $\mathrm{OdB}$ & -28.20 & -28.38 & -29.58 & -29.69 & -30.25 & -30.39 \\
\hline $2 \mathrm{~dB}$ & -31.84 & -32.03 & -33.29 & -33.41 & -33.94 & -34.10 \\
\hline $4 d B$ & -35.39 & -35.57 & -36.78 & -36.90 & -37.36 & -37.53 \\
\hline
\end{tabular}

code's effects promoting the $3^{\text {rd }}$ medium energy sub-constellation (see constellation mapping in Fig. 5). As we can see, there is an increase in the number of medium symbols of this sub-constellation during transmission. So, the shaping code allows a change in the constellation and improves the decision process during decoding.

In Table 5, we give the upper and lower ACPR values of the PA output for different input power such as 


$$
\operatorname{ACPR}_{\mathrm{dB}}^{R}=10 \log _{10}\left(\frac{\sum_{\omega \in R}|Y(\omega)|^{2}}{\sum_{\omega \in M_{c}}|Y(\omega)|^{2}}\right)
$$

where $R$ denotes the lower (noted $\mathrm{L}$ ) or upper (noted $\mathrm{U}$ ) first adjacent channel frequencies of $20 \mathrm{MHz}$ bandwidth. $M_{c}$ is the primary channel frequencies, $Y(\omega)$ is the DFT of the output signal.

For different IBO values, we can note a reduction of about $2 \mathrm{~dB}$ by applying the proposed method, compared to the original signal, this is mainly the effect of the DCT method, known for its good frequency compaction and spectral efficiency [36]. Combined with the shaping technique, it allows significant PAPR reduction while maintaining the spectrum in the standard specification.

We can conclude that applying the proposed method makes it possible to reduce the fluctuations of the signal and consequently reduce the nonlinear distortions generated by the power amplifier.

\subsection{Experimental validation on $20 \mathrm{~W}$ power amplifier}

In order to validate the effectiveness of the proposed method, experimental tests have been carried out using a 20W test bench Fig. 17. The IQ baseband signals are generated and uploaded from the PC Workstation to the RF Agilent N5182A MXG Vector Signal Generator. The stimulus signal is an OFDM signal in WLAN 802.11y standard, 16-QAM modulation with $20 \mathrm{MHz}$ bandwidth for experiments. Output signal is injected to a twostages RF PA 20W class-AB 3.7 GHz in LDMOS technology from NXP society, operating in the 3.4-3.8 GHz range. Output signals are measured in an RF format with a four-channel oscilloscope at a sampling rate of $40 \mathrm{GHz}$ (LeCroy SDA820Zi-A) and an Agilent N9020A MXA X-Series Signal Analyzer. All equipment communicates through Local Area Network connector (LAN).

In Fig. 18, we present the gain (AM/AM) and phase (AM/PM) characteristics of the used PA, defined, respectively, by the output signal magnitude and phase, for a $3.7 \mathrm{GHz}$ carrier frequency. From the amplitude conversion (blue curve), we can see the linear, compression and saturation regions. We can also define the $1 \mathrm{~dB}$ compression point corresponding to an input power of $12 \mathrm{dBm}$. As in Sect. 3.1, this point is taken as reference for OdB of IBO.

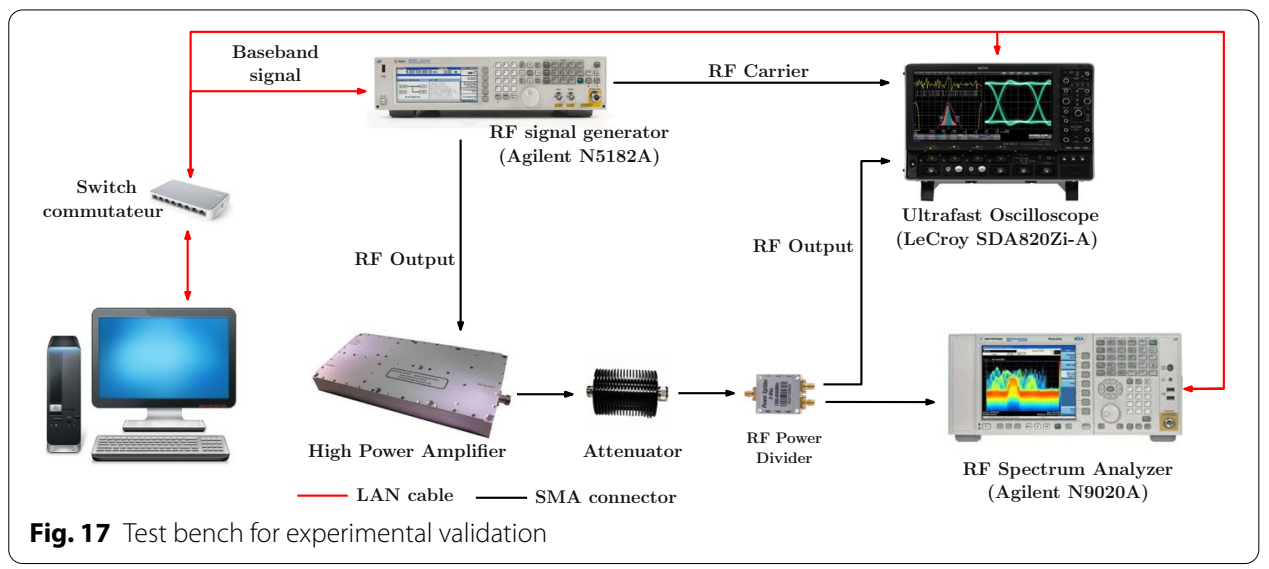




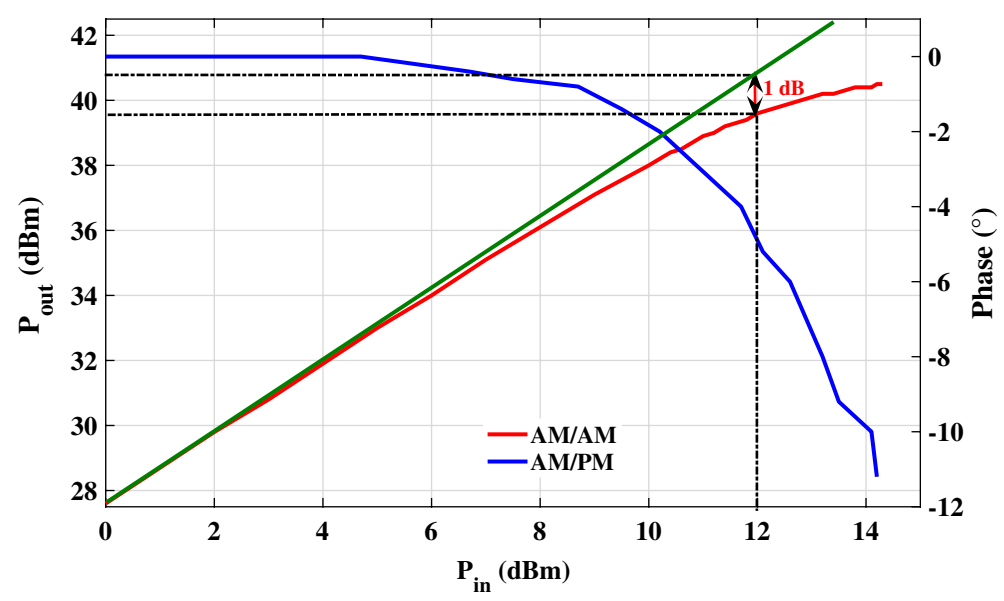

Fig. 18 AM/AM and AM/PM characteristics of the used PA

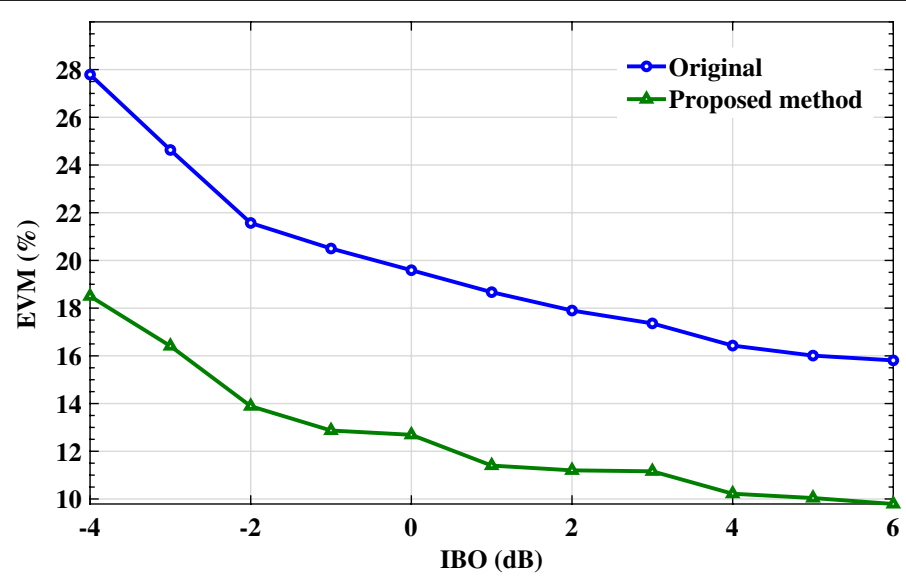

Fig. 19 Measured EVM versus IBO

\subsubsection{Experimental results}

To evaluate the effectiveness of the proposed method, we show in Figs. 19, 20 the EVM and BER results with and without the PAPR reduction.

For the overall input power range, the proposed method improves the transmission quality with a decrease in the EVM and the BER over PA nonlinearities. If we operate in compression region (at OdB of IBO for example), the improvement in EVM is up to 6\%.

The BER reaches $4 \cdot 10^{-3}$ compared to $10^{-2}$ for the PA without PAPR reduction. Also, if the operating point is chosen in the linear region (at IBO $=6 \mathrm{~dB}$ for example), we can see an improvement of EVM up to $8 \%$ and by a factor of 6 to 19 for the BER.

Figure 21 presents the symbols distortion with and without PAPR reduction for an IBO of $-2 \mathrm{~dB}$, i.e., PA operating in saturation. As we can see, the proposed method reduces the dispersion on the constellation points and makes easier the decoding process from mapping symbols to a binary stream.

The PAPR reduction methods can be evaluated according to their ability to respect the specification criteria like the used standard's frequency mask. Figure 22 addresses this 


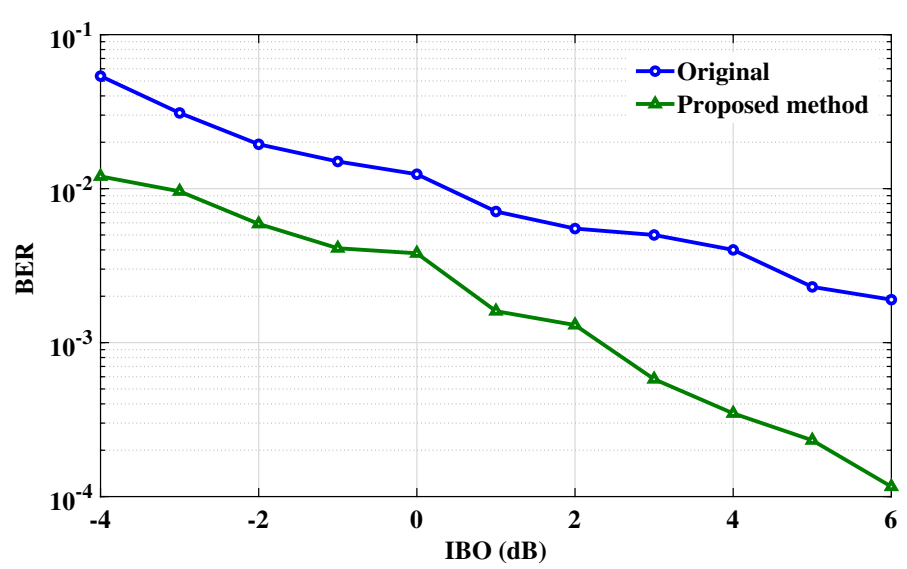

Fig. 20 Measured BER versus IBO
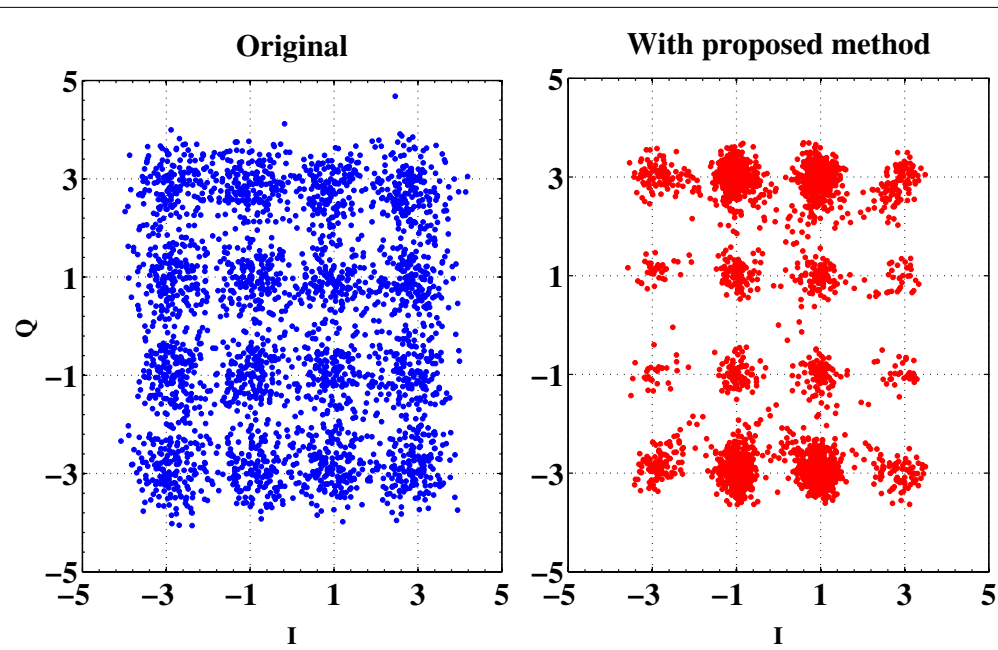

Fig. 21 Measured constellation diagrams

issue and illustrates the proposed method's impact on the frequency response at IBO $=-2 \mathrm{~dB}$. As we can see, the PA introduces additional components in the adjacent bands due to its high nonlinearities in the studied region. However, the proposed method allows a reduction of the out of band spurious and maintains the output spectrum in the specification mask of the defined standard.

\subsubsection{Comparative study}

Table 6 presents the proposed technique performances compared to the TR, DCT and shaping techniques [34,37]. This study is performed using essential criteria like PAPR reduction, the increase in the average power and the power efficiency enhancement. Notice that the power efficiency enhancement results are obtained using the efficiency curves versus input power of the used PA.

We can observe that the TR presents the best improvement in term of PAPR reduction. Like other techniques based on adding signal (see Table 1), the main drawback is 


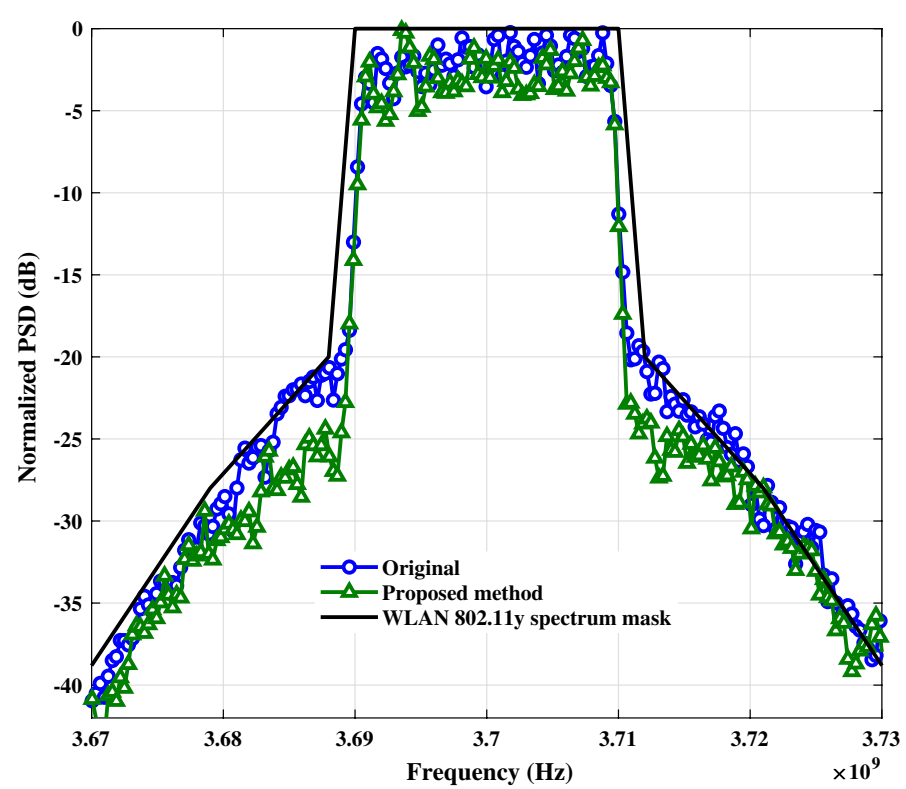

Fig. 22 Impact of nonlinear PA model on frequency responses with the proposed method $(I B O=-2 d B)$

Table 6 Comparative study

\begin{tabular}{llll}
\hline & \multicolumn{2}{l}{ Performance effects } & \\
\cline { 2 - 4 } & PAPR & Average & Power efficiency \\
power & improvement (\%)
\end{tabular}

an increase in the average power, around $0.9 \mathrm{~dB}$. Also, Shaping code and the DCT are complementary in their results with a better reduction of the average power for the first one due to the mapped digital symbols' concentration on the low energy sub-constellation [sub-constellation (1) in Fig. 5]. The proposed method combines their performances and ensures a trade-off between reducing the PAPR and increasing the average power. Note that the power efficiency of the proposed method is greater than that of the TR method [37]. However, it is worth mentioning that it introduces minor modifications in the system's coding and decoding schemes, making it not fully downward compatibility, unlike the TR.

\section{Conclusions}

In this paper, we proposed and applied a PAPR reduction method for multicarrier systems based on the conjunction of the DCT and the shaping technique. This study shows the existence of shaping codes to simultaneously reduce the PAPR and out-of-band interferences, by giving priority to low and medium energy symbols. We also showed that the combined scheme (DCT + shaping code) provides better performances than 
each technique alone, by combining their benefits. In this cascaded structure, the DCT block allows an improvement of ACPR, even in the presence of nonlinear PA with memory and a noisy channel.

The cascaded method usefulness is illustrated by nonlinear simulations using a memory polynomial model and a noisy channel with different SNR and IBO. From these results, we observed an improvement in EVM and BER of about $7.5 \%$ and one decade and a half, respectively. In this case, the power amplifier can be used in its nonlinear region $(-2 \mathrm{~dB}$ of $\mathrm{IBO})$, with better efficiency and without loss in quality of service.

The experimental results on a $20 \mathrm{~W}-3.7 \mathrm{GHz}$ class $\mathrm{AB}$ power amplifier show also that the proposed method can significantly improve the system performances (EVM and BER) and attenuate the PA undesirable consequences on adjacent channel regrowth. From these results, we observed an EVM improvement of 6 to $8 \%$ and about one decade on the BER. We can conclude that the new technique is a good candidate to enhance power efficiency on RF transmitters for a given distortion level.

\begin{abstract}
Abbreviations
PA: Power Amplifier; PAPR: Peak-to-Average Power Ratio; DCT: Discrete Cosine Transform; OFDM: Orthogonal Frequency Division Multiplex; RF-PA: Radio Frequency-Power Amplifier; BER: Bit Error Rate; EVM: Error Vector Magnitude; WLAN: Wireless Local Area Network; ACE: Active Constellation Extension; TR: Tone Reservation; SLM: Selected Mapping; PTS: Partial Transmit Sequence; IFFT: Inverse Fast Fourier Transform; QAM: Quadrature Amplitude Modulation; DFT: Discrete Fourier's Transform; DCT-I: Uni-dimensional DCT; DCT-II: Two-dimensional DCT; DCT-III:Thirds-dimensional DCT; IDCT: Inverse Discrete Cosine Transform; UL: Up-Link; DL: Down-Link; CCDF: Complementary Cumulative Distribution Function; AWGN: Additive White Gaussian Noise; SNR: Signal-to-Noise Ratio; S.C: Shaping Code; LSB: Least Significant Bits; MSB: Most Significant Bits; LLR: Logarithm Likelihood Ratio; ACPR: Adjacent Channel Power Ratio; AM/AM: Amplitude to Amplitude Modulation; AM/PM: Amplitude to Phase Modulation; IBO: Input Back-Off; QoS: Quality of Service; LAN: Local Area Network.
\end{abstract}

\title{
Acknowledgements
}

This work is the result of a scientific cooperation and the authors wish to acknowledge the support of PROFAS B+ and the Hubert Curien Partnership (PHC-TASSILI).

\section{Authors' contributions}

All authors have contributed to the analytic and numerical results. The authors read and approved the final manuscript.

Availability of data and materials

The authors declare that all the data and materials in this manuscript are available from the authors.

\section{Competing interests}

The authors declare that they have no competing interests.

\section{Author details}

'Laboratory Technology of Communication, Faculty of Technology, University of Saida, BP 138 En-Nasr, 20000 Saida, Algeria. ${ }^{2}$ XLIM Laboratory UMR-CNRS 7252, Institute of Technology, Angoulême-University of Poitiers, 4 avenue de Varsovie, 16021 Angoulême, France.

Received: 23 July 2020 Accepted: 11 February 2021

Published online: 23 February 2021

\section{References}

1. M.-O. Pun, M. Morelli, C.-C.J. Kuo, Multi-Carrier Techniques for Broadband Wireless Communications: A Signal Processing Perspective (Imperial College Press, London, 2007)

2. R. Prasad, OFDM for Wireless Communications Systems, Artech House (2004)

3. T. Jiang, Y. Wu, An overview: peak-to-average power ratio reduction techniques for OFDM signals. IEEE Trans. Broadcast. 54, 257-268 (2008)

4. D. Guel, J. Palicot, Clipping formulated as an adding signal technique for OFDM peak power reduction, in VTC Spring 2009-IEEE 69th Vehicular Technology Conference (2009), pp. 1-5

5. R. Saroj, A cooperative additional hybrid and clipping technique for PAPR reduction in OFDM system, in 5th International Conference on Eco-friendly Computing and Communication Systems (ICECCS) (2016), pp. 53-57

6. M.J. Azizipour, K. Mohamed-Pour, Clipping noise estimation in uniform tone reservation scenario using OMP algorithm, in 8th International Symposium on Telecommunications (IST) (2016), pp. 500-505 
7. Hu. Su, W. Gang, Wen Qingsong, Y. Xiao, L. Shaogian, Nonlinearity reduction by tone reservation with null subcarriers for WiMAX system. Wireless Pers. Commun. 54(2), 289-305 (2010)

8. Z. Zheng, G. Li, An efficient FPGA design and performance testing of the ACE algorithm for PAPR reduction in DVBT2 systems. IEEE Trans. Broadcast. 1, 134-143 (2017)

9. R. Saroj, Performance evaluation of hybrid ACE-PTS PAPR reduction techniques, in 11th International Conference on Computer Engineering Systems (ICCES) (2016), pp. 407-413

10. J. Tellado-Mourelo, Peak to Average Power Ratio Reduction for Multicarrier Modulation. PhD thesis, Stanford University (1999)

11. O.A. Gouba, Y. Louet, Adding signal for peak-to-average power reduction and predistortion in an orthogonal frequency division multiplexing context. IET Signal Proc. 7(9), 879-887 (2013)

12. S. Hussain, Y. Louet, J. Palicot, Peak power control in cognitive radio context. IET Commun. 6(8), 861-871 (2012)

13. H. Sohtsinda, S. Bachir, C. Perrine, C. Duvanaud, An evaluation of hybrid tone reservation method for PAPR reduction using power amplifier with memory effects, in IEEE International Conference on Electronics, Circuits and Systems (ICECS) (2016), pp. 676-679

14. R.-W. Bauml, R.-F.-H. Fischer, J.-B. Huber, Reducing the peak-to-average power ratio of multicarrier modulation by selected mapping. Electron. Lett. 32, 2056-2057 (1996)

15. J. Ji, G. Ren, H. Zhang, A semi-blind SLM scheme for PAPR reduction in OFDM systems with low-complexity transceiver. IEEE Trans. Veh. Technol. 64, 2698-2703 (2015)

16. M.. -S. Hossain, T. Shimamura, Low-complexity null subcarrier-assisted OFDM PAPR reduction with improved BER. IEEE Commun. Lett. 21, 2249-2252 (2016)

17. A... -E. Jones, T.. -A. Wilkinson, S.. -K. Barton, Block coding scheme for reduction of peak to mean envelope power ratio of multicarrier transmission schemes. Electron. Lett. 30, 2098-2099 (1994)

18. H. Ochiai, A new trellis shaping design for OFDM, in The 5th International Symposium on Wireless Personal Multimedia Communications, vol. 3 (2002), pp. 1019-1023

19. S.. -H. Muller, J.. -B. Huber, OFDM with reduced peak-to-average power ratio by optimum combination of partial transmit sequences. Electron. Lett. 33, 368-369 (1997)

20. A. Hanprasitkum, A. Numsomran, P. Boonsrimuang, Improved PTS method with new weighting factor technique for FBMC-OQAM systems, in 19th International Conference on Advanced Communication Technology (ICACT) (2017), pp. 143-147

21. Y. Aimer, B.S. Bouazza, S. Bachir, C. Duvanaud, PAPR reduction performance in WIMAX OFDM systems using interleavers with downward-compatibility, in 2018 IEEE International Symposium on Circuits and Systems (ISCAS) (2018), pp. 1-5

22. Y. Aimer, B.S. Bouazza, S. Bachir, C. Duvanaud, Interleaving technique implementation to reduce PAPR of OFDM signal in presence of nonlinear amplification with memory. J. Telecommun. Inf. Technol. 54, 14-22 (2018)

23. G. Pepin Magnangana Zoko, M. Ibrahim James, A. Sroy, SLM using riemann sequence combined with DCT transform for PAPR reduction in OFDM communication systems. in World Academy of Science, Engineering and Technology, International Journal of Information and Communication Engineering, vol. 6(4) (2012), pp. 394-399

24. M.R. Hossain, K.T. Ahmmed, Non-linear companding based hybrid PAPR reduction approach for DCT-SCFDMA system. IET Commun. 12(12), 1468-1476 (2018)

25. Z. Kai, Z. Jian, X. Liyong, A research on improving the performance of OFDMA system by using DCT/IFFT structure, in 2nd International Conference on Information Science and Engineering (ICISE) (2010), pp. 4-6

26. J. Palicot, Radio Engineering: From Software Radio to Cognitive Radio (John Wiley and Sons, Hoboken, 2013).

27. B.S. Bouazza, Y.S. Le Goff, A. Garadi, C. Perrine, R. Vauzelle, A novel constellation shaping technique for bit-interleaved coded modulation. Wirel. Pers. Commun. 71(2), 519-528 (2013)

28. R. Van Nee, R. Prasad, OFDM for Wireless Multimedia Communications. Artech House (2000)

29. Y.S. Le Goff, S. Bayan Sharif, A. Shihab Jimaa, A new bit-interleaved coded modulation scheme using shaping coding, IEEE Global Telecommunications Conference, GLOBECOM '04 (2004), pp. 1-4

30. W. Burger, M.J. BurgePun, Digital Image Processing: An Algorithmic Introduction Using Java (Springer, London, 2008).

31. J. Makhoul, A fast cosine transform in one and two dimensions. IEEE Trans. Acoustics Speech Signal Process. 28(1), 27-34 (1980)

32. N. Ahmed, T. Natarajan, K.R. Rao, Discrete cosine transform. IEEE Trans. Comput. C23(1), 90-93 (1974)

33. D.M. Tan, H.R. Wu, Multi-dimensional discrete cosine transform for image compression, ICECS'99. Proceedings of ICECS '99. 6th IEEE International Conference on Electronics, Circuits and Systems (1999)

34. Y. Aimer, 'Étude des performances d'un système de communication sans fil à haut débit. PhD thesis, University of Poitiers. https://hal.archives-ouvertes.fr/tel-02486765 (2019)

35. J.Wood, Behavioral Modeling and Linearization of RF Power Amplifiers. Artech House (2014)

36. F.S. Al-Kamal, E.S. Hassan, M.A. El-Naby et al., An efficient transceiver scheme for SC-FDMA systems based on discrete wavelet transform and discrete cosine transform. Wireless Pers. Commun. 83(4), 3133-3155 (2015)

37. B. Koussa, Optimisation des performances d'un système de transmission multimédia sans fil basé sur la réduction du PAPR dans des configurations réalistes. PhD thesis, Université de Poitiers, Avril (2014)

\section{Publisher's Note}

Springer Nature remains neutral with regard to jurisdictional claims in published maps and institutional affiliations. 